\title{
Solving Impulsive Control Problems by Discrete-Time Dynamic Optimization Methods ${ }^{1}$
}

\author{
R.T.N. CARDOSO ${ }^{2}$, Department of Electrical Engineering, Universidade Federal \\ de Minas Gerais, 30123-970 Belo Horizonte, MG, Brazil
}

R.H.C. TAKAHASHI, Department of Mathematics, Universidade Federal de Minas Gerais, 30123-970 Belo Horizonte, MG, Brazil.

\begin{abstract}
This work presents an open-loop discrete-time dynamic optimization scheme for continuous-variable impulsive control problems. This methodology can be more useful than the classical optimal control in several contexts, since it delivers control actions just in discrete times, which introduce discontinuities in the system state variables. Two case studies are presented: the biological control of pests in crops using a prey-predator model and the optimal vaccination in epidemics control using an SIR model.
\end{abstract}

Keywords. Impulsive Control, Dynamic Programming, Prey-predator, SIR.

\section{Introduction}

Control actions in the form of jumps at certain time instants can be useful to be considered in many classes of continuous-time control problems coming from real world applications. This technique is called impulsive control. The main idea is to split the continuous-time interval in some stages, performing control actions impulsively just in some time instants. The dynamic system keeps its autonomous dynamics in the time intervals between the consecutive impulsive control actions. The concept and the principles of the impulsive control, and also some simple examples of applications in systems whose variables should be changed instantaneously have been presented by Yang [14].

The article [6] has used continuous-time dynamic programming to solve an impulsive control problem, in which solutions have been synthesized via the Hamilton-Jacobi-Bellman (HJB) approach. Discrete-time dynamic programming with continuous-variable discretization is a well-known multi-stage optimization technique [1], that could be another alternative for solving the same problem. In the present article, the solution of impulsive control problems is sought by means of an open-loop continuous-variable dynamic optimization algorithm. The scheme

\footnotetext{
${ }^{1}$ The authors would like to thank the Brazilian agencies, CAPES and CNPq for the financial support. They would also thank to Andre R. Cruz.

${ }^{2}$ rodrigoc@cpdee.ufmg.br

3 taka@mat.ufmg.br
} 
proposed here is more flexible than the HJB approach, since it allows rather arbitrary objective function and constraints, and is an alternative to using enumerative discrete-time discrete-variable dynamic programming algorithms, which have prohibitive computational complexity $[1,3]$.

Two case studies are considered here: the biological control of pests in a farm using a prey-predator model and the pulse vaccination strategy using an SIR epidemic model. Some recent applications of impulsive control in prey-predator models and in SIR epidemic models, for example, are presented in $[7,11]$.

This article is structured as follows: Section 2. discusses the impulsive control. Section 3. discusses the proposed open-loop discrete-time approach for impulsive control problems. Section 4. presents the application of the proposed methodology in two numerical case studies.

\section{Impulsive Control}

Let $T \in \mathbb{R}$ be the optimization horizon and $\Gamma=\left\{\tau_{0}, \ldots, \tau_{N}\right\}$ be a set of control instants in $[0, T]$, such that: $\tau_{k}<\tau_{k+1}, \tau_{0}=0$ and $\tau_{N}=T$. These intervals do not have to be equidistant. Consider a plant whose state variables belong to $X \subset \mathbb{R}^{n}$ and the inputs or the control laws belong to $U_{k} \subset \mathbb{R}^{m}$, for all $k=0, \ldots, N-1$. The state at time $\tau_{k}$ is $x\left(\tau_{k}\right)$ (a continuous-time variable) and the control action at time $\tau_{k}$ is $u[k]$ (a discrete-time variable). The time instant $\tau_{k}^{+}$is defined as a time instant "just after" the impulsive action in $\tau_{k}$. The time instant $\tau_{k}^{+}$is formally defined as a number that fulfills $\tau_{k}^{+}=\tau_{k}+\epsilon$, with $0<\epsilon<\delta$, for any finite $\delta>0$.

Definition 2.1 (Yang). In an impulsive control problem, the state at each time $\tau_{k}, x\left(\tau_{k}\right) \in X$, can be changed impulsively by

$$
x\left(\tau_{k}^{+}\right)=x\left(\tau_{k}\right)+u[k],
$$

with $u[k] \in U_{k}$.

The impulsive differential equations (IDEs) describing the resulting dynamic system can have the form:

$$
\left\{\begin{array}{l}
x^{\prime}(t)=f_{1}(t, x(t)) \\
t \in\left(\tau_{k}^{+}, \tau_{k+1}\right] \\
x\left(\tau_{k}^{+}\right)=x\left(\tau_{k}\right)+u[k] \\
k=0, \ldots, N-1
\end{array}\right.
$$

where $f_{1}: \mathbb{R}_{+} \times \mathbb{R}^{n} \rightarrow \mathbb{R}^{n}$ is continuous.

Each initial value problem in (2.2) is valid when there is no control action the system presents its autonomous dynamics within such time intervals. A new initial condition for the system, $x\left(\tau_{k}^{+}\right)$, is established in each stage according to the difference equation (2.1), linking each stage with the next one. The equations (2.2) describe a hybrid system, in which a continuous-time dynamics is driven, at discrete instants, by impulsive control inputs that cause discontinuities in the system state variables. Such hybrid system involves the interaction of a continuous-time 
and a discrete-time dynamics: each discrete-time action performs jumps on the continuous-time system, and the continuous dynamics performs the state transition of the discrete dynamics. Hybrid systems with impulsive control have been studied in $[2,10,13]$, among others.

The aim of this article is to study impulsive control problems from a discrete-time optimization point of view. For this, the following discrete-time variable notation is considered: $x[k]=x\left(\tau_{k}\right)$ and $x\left[k^{+}\right]=x\left(\tau_{k}^{+}\right)$. Thus, the corresponding discrete-time dynamic system can be written as

$$
x\left[k^{+}\right]=x[k]+u[k] .
$$

In this sense, the values of the state variables are considered in the optimization just in a time instant set $\Gamma=\left\{\tau_{0}, \ldots, \tau_{N}\right\}$ previously chosen, and the state in each stage is supposed to be calculated by the impulsive differential equation shown in (2.2). In many control problems, there is an end-point constraint: the final state must reach a target, for all feasible initial state:

$$
x(T)=x^{*} .
$$

This end-point target may be an equilibrium of the impulsive system. An equilibrium $\bar{x}$ of an impulsive dynamic system is defined as a vector for which holds:

$$
\bar{x}=x\left(\tau_{k}\right)=x\left(\tau_{k+1}\right), \quad \forall k .
$$

Thus, considering a fixed time interval associated with this equilibrium $\bar{x}$, there are two other fixed variables: the control $\bar{u}$, such that: $\bar{u}=u[k]=u[k+1]$, for each $k$, and $\bar{x}^{+}$, the initial condition of the IVP, such that: $\bar{x}^{+}=x\left(\tau_{k}^{+}\right)=x\left(\tau_{k+1}^{+}\right)$, for each $k$. For this situation of stability, $\bar{x}^{+}=\bar{x}+\bar{u}$.

\section{Discrete-time Dynamic Optimization Scheme}

Consider $g_{k}(x[k], u[k])$ as the separable cost function of the time-stage $k$, and $g_{N}(x[N])$ as the final cost. A ranking based on the sum of the costs per stage leads to the optimal solution. This is the "Bellman's Optimality Principle" [1].

The discrete-time dynamic optimization problem for impulsive systems is formulated as

$$
\min _{u[0], \ldots, u[N-1]} \sum_{k=0}^{N-1} g_{k}(x[k], u[k])+g_{N}(x[N])
$$

subject to:

$$
\left\{\begin{array}{l}
x^{\prime}(t)=f_{1}(t, x(t)) \\
t \in\left(\tau_{k}^{+}, \tau_{k+1}\right] \\
x\left(\tau_{k}^{+}\right)=x\left[k^{+}\right]=x[k]+u[k] \\
k=0,1, \ldots, N-1 ; \\
x[0]=x_{0} \text { and } x[N]=x^{*} \text { are given. }
\end{array}\right.
$$


Due to the difficulties to reach on equality constraints, a relaxation in the endpoint target (2.4) can be performed, using an inequality constraint defining a set of admissible size around the target, for instance a ball with a given radius $\epsilon$ around the end-point target.

The impulsive problems are treated in this article as continuous-variable discretetime dynamic optimization problems. The optimization procedure employed here is similar to the one presented in [3], which performs an optimization in open-loop, considering the objective function and all the constraints as functions of the initial state and of the optimal control action sequence only. This technique is similar to the sub-optimal procedure known as open-loop feedback controller (OLFC) [1].

The idea is study the dynamics of the system through the iteration of sets instead of either using discretization and enumerative methods that place the dynamics in arcs of a graph (as in discrete-variable dynamic programing), or generating analytical solutions (as in variational formulations of continuous variable problems). It is well-known that enumerative algorithms have hard computational costs [1] and that variational formulations usually involve analytical manipulations that require that the objective function and constraints have some specific mathematical forms [4]. The proposed dynamic optimization scheme for impulsive control involves: (i) To allow the relaxation of the end-point constraint at the final stage; (ii) To consider the pre-image of the relaxed end-point into the initial stage, re-writing the states as functions of the initial state and of the optimal control action sequence; (iii) To make the dynamic optimization sequentially, for each stage, updating the initial state, running the open-loop optimization procedure, and implementing just the present-time control.

\section{Numerical Case Studies}

\subsection{Biological control of pests}

Biological control of pests in crops aim the reduction of pest populations, achieved with the action of other living organisms, often called natural enemies $[5,8]$. The interaction of pests and their natural enemies (organisms that can cause injury to the pests, such as predators and parasites) can be represented by predator-prey models. This article considers the prey-predator model shown in (4.1).

$$
\left\{\begin{array}{l}
x^{\prime}(t)=x(a-\gamma x-\alpha y), \\
y^{\prime}(t)=y(-b+\beta x) .
\end{array}\right.
$$

Above, the parameters $a, \gamma, \alpha, b$ and $\beta$ are positive constants with known values and $x=x(t)$ and $y=y(t)$ denote the density of preys and predators, respectively.

This case study considers the relation between the caterpillar of the soy (Anticarsia gematalis) and its enemies, like wasps and spiders. The values of the system parameters, borrowed from [8], are shown in Table 1.

The origin $(0,0)$ is an unstable equilibrium of this system and there is a stable equilibrium with nonzero number of pests $(65.5172,4.7241)$. As the acceptable number of pests is $x^{*}=20$, a control action must be applied, through the insertion 
Table 1: Parameter values of the prey-predator system (4.1) considering the relation between the Anticarsia gematalis and its enemies.

\begin{tabular}{|c|c|c|c|c|}
\hline$a$ & $b$ & $\alpha$ & $\gamma$ & $\beta$ \\
\hline 0.16 & 0.19 & 0.02 & 0.001 & 0.0029 \\
\hline
\end{tabular}

of caterpillar natural enemies. The work [8] has studied this control problem, seeking solutions by continuous-time dynamic programming.

The objective function of this problem is supposed to be linear, with the parameters $c_{k}$ (corresponding to the pests) and $d_{k}$ (corresponding to the predators) as positive constants. This assumption is reasonable, since the cost of predators is proportional to the quantity being launched, and the cost of crop loss is proportional to the number of pest units (at least within a large variable range, in which the soy plants do not approximate a situation of being destroyed). The decision variable sequence $\{u[0], \ldots, u[N-1]\}$ represents the density of predators to be launched at each discrete time stage.

This impulsive-time dynamic optimization problem can be formulated as

$$
\min _{u[0], \ldots, u[N-1]} \sum_{k=0}^{N-1}\left(c_{k} x[k]+d_{k} u[k]\right)+c_{N} x[N]
$$

subject to:

$$
\left\{\begin{array}{l}
x^{\prime}(t)=x(a-\gamma x-\alpha y) \\
y^{\prime}(t)=y(-b+\beta x) \\
t \in\left(\tau_{k}^{+}, \tau_{k+1}\right] \\
x\left(\tau_{k}^{+}\right)=x\left[k^{+}\right]=x[k] \\
y\left(\tau_{k}^{+}\right)=y\left[k^{+}\right]=y[k]+u[k] \\
k=0,1, \ldots, N-1 \\
x[0]=x_{0} \text { and } y[0]=y_{0} \text { are given; } \\
x[N] \leq x^{*}=20
\end{array}\right.
$$

The initial prey density is $x_{0}=100$, the initial predator density is $y_{0}=0$ and the time horizon is $T=200$ days. The duration of each stage is $\delta T=20$ days; therefore, there are $N=10$ stages. The constants $c_{k}$ and $d_{k}$ are all equal to 1 .

Figure 1 shows the optimal evolution of preys and predators throughout the stages (in logarithmic scale) and its phase diagram. The cost function value of this solution is $J^{*}=446$. Notice that the end-point $x^{*}$ has been reached since the first optimization stage. A direct comparison between the proposed strategy and the continuous-time control action proposed in [8] cannot be made, since in [8] the resulting control action is continuous (the release of predators is assumed to be performed continuously along the time), which is very impractical. However, the control action obtained from [8] can be discretized in the following way: (i) some time instants are defined for performing the control action; (ii) the integral of the continuous control action is applied, impulsively, in such time instants. In this case, 
the value of the cost function value that is achieved becomes $J^{*}=1177.6$, much greater than the one that is achieved with the methodology proposed here.

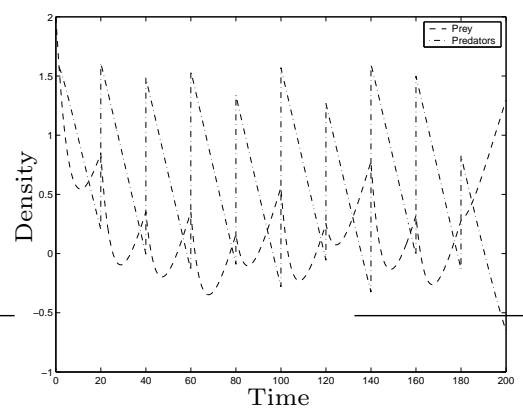

(a) Time-evolution

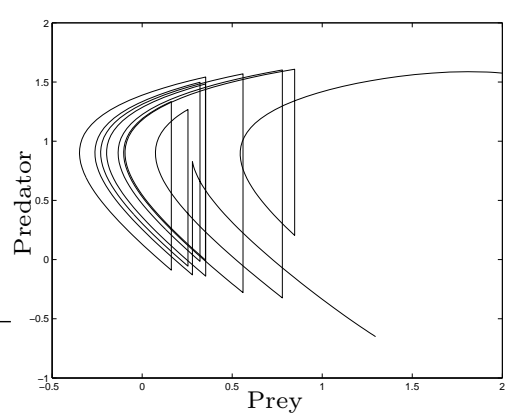

(b) Phase diagram

Figure 1: Behavior of the predator-prey system (4.1) with the optimal impulsive control action. Due of the logarithmic scale, the null initial condition is not displayed in this figure.

\subsection{Optimal vaccination strategies in epidemic control}

Epidemic is an alteration in one or more characteristics in a significant number of individuals, normally related to the the loss of health. The interaction between individuals of a population and the environment is the basic mechanism behind the epidemic spread. These interactions have been mathematically modeled and studied. This case study considers the mathematical model of an epidemic propagation called SIR [9,12]. This model classifies the individuals in three states: susceptible $(S)$, infected $(I)$ and recovered $(R)$. These three states are related by the dynamic system:

$$
\left\{\begin{array}{l}
S^{\prime}(t)=\mu N-\mu S-\beta I S / N \\
I^{\prime}(t)=\beta I S / N-\gamma I-\mu I \\
R^{\prime}(t)=\beta I-\mu R .
\end{array}\right.
$$

Above, $N$ is the size of population, $\mu$ is the ratio of new susceptible per time unit, $\gamma$ is the ratio of infected individuals that are recovered per time unit and $\beta$ is the ratio of contacts between individuals that lead to disease transmission, per time unit. It is assumed that $N$ is constant along the time; therefore the variables can be written as ratios: $s(t)=S(t) / N, i(t)=I(t) / N, r(t)=R(t) / N$ and also $r(t)=1-s(t)-i(t)$. Thus, the dynamic system (4.3) can be rewritten as a two-dimensional system:

$$
\left\{\begin{array}{l}
s^{\prime}(t)=\mu-\mu s-\beta i s \\
i^{\prime}(t)=\beta i s-\gamma i-\mu i
\end{array}\right.
$$

The basic reproductive number $R_{0}$ is defined as $R_{0}=\beta /(\mu+\gamma)$, which represents the average number of infections produced by an infected individual. It can be shown [9] that the system (4.4) has an asymptotically stable endemic equilibrium 
iff $R_{0}>1$. Table 2 presents the values of the parameters in this paper, borrowed from [12], which are related to measles epidemics. Note that $R_{0}>1$, thus this system has an asymptotically stable endemic equilibrium. In fact, by simulation, it can be seen that $24 \%$ of the population would be infected in the equilibrium state. Therefore, a control action must be applied in this system, for instance a vaccination process. This work proposes using non-fixed pulse vaccination strategies, which is an impulsive control defined by repeated application of vaccination in discrete times with constant time interval. Pulse vaccination has been studied recently also in [7].

Table 2: Parameter values of the system SIR (4.4) considering the epidemic of measles in the United Kingdom in 1950-68.

\begin{tabular}{|c|c|c|c|c|}
\hline$\mu$ & $\gamma$ & $\beta$ & $N$ & $R_{0}$ \\
\hline $1 / 70$ & $1 / 24$ & 0.95 & $2 \times 10^{6}$ & 17 \\
\hline
\end{tabular}

The strategy to be considered here is: the public health policy-maker should choose a time-interval for vaccination and an equilibrium point (in the sense of impulsive system equilibrium), with an acceptable level of infected individuals ${ }^{4}$. This equilibrium corresponds to a constant percentage of the population being vaccinated in each time-stage. This choice can be performed via a bi-objective analysis, considering both the cost related to the damage with infection and the vaccination cost as a non-linear function of the vaccination coverage. The percentage of the population to be vaccinated in the infinite time horizon, $\bar{p}$, is associated to constant numbers of susceptible and infected (those will be called $\bar{s}$ and $\bar{i}$, respectively). The vector of the equilibrium state of the impulsive system is taken as the end-point constraint of the dynamic optimization problem.

The decision variable sequence is $\{p[0], \ldots, p[N-1]\}$, the percentage of susceptible to be vaccinated in each stage $(k)$. A positive-definite quadratic cost function is considered (the parameters $c_{k}$ and $d_{k}$ are positive scalars). The end-point constraint is $i[N]=i^{*}$, corresponding to the given impulsive equilibrium $\bar{p}$. Note that the optimization with the equality end-point constraint $i[N]=i^{*}$ may be difficult to be attained, and a relaxation in the end-point constraint is allowed. An impulsive control formulation considering a square-ball (without lost of generality) around the target can be given as

$$
\min _{p[0], \ldots, p[N-1]} \sum_{k=0}^{N-1}\left(c_{k} i[k]^{2}+d_{k} p[k]^{2}\right)+c_{N} i[N]^{2}
$$

\footnotetext{
${ }^{4}$ For some diseases, the acceptable level should be zero infected individuals, corresponding to the disease eradication.
} 
subject to:

$$
\left\{\begin{array}{l}
s^{\prime}(t)=\mu-\mu s-\beta i s \\
i^{\prime}(t)=\beta i s-\gamma i-\mu i ; \\
t \in\left(\tau_{k}^{+}, \tau_{k+1}\right] \\
s\left(\tau_{k}^{+}\right)=s\left[k^{+}\right]=s[k](1-p[k]) ; \\
s[k]=s\left(\tau_{k}\right) ; \\
i\left(\tau_{k}^{+}\right)=i\left[k^{+}\right]=i[k] ; \\
i[k]=i\left(\tau_{k}\right) ; \\
k=0,1, \ldots, N-1 ; \\
s[0]=s_{0} \text { and } i[0]=i_{0} \text { are given; } \\
\left\|i[N]-i^{*}\right\|_{\infty} \leq \epsilon .
\end{array}\right.
$$

The initial susceptible ratio is $s_{0}=0.999$, the initial infected ratio is $i_{0}=0.001$ and the optimization horizon is $T=100$ years. The duration of each stage is one year, $\delta T=1$. Therefore, there are $N=100$ stages. The end-point target of this problem is $i^{*}=10^{-5}$, corresponding to the constant periodic impulsive vaccination with $\bar{p}=0.5$. The ratio of the square-ball around the chosen end-point target is supposed to be $\epsilon=10^{-4}$, what means that it allows $0.1 \%$ of infected individuals in the final stage. The constant parameters of the objective function are $c_{k}=0,9 \times 10^{7}$ and $d_{k}=0,1 \times 10$. Each decision variable $p[k]$ is constrained to be between 0.4 and 0.8 (since the vaccination of more than $80 \%$ of a population can be very difficult).

An interesting observation, related to the relaxation of the end-point constraint in this case is: the SIR model assumes a population of infinite size, in which the statistical fluctuations around the mean predicted behavior do not occur. As long as the actual populations are finite, a very low but non-zero level of infected individuals will eventually lead to disease eradication, due to such fluctuations. Exploiting such effect can lead to disease eradication policies that are much less costly than "bruteforce" ones, with similar effectiveness.

Figure 2 shows the evolution of the system (in logarithmic scale) and its phase diagram for a constant periodic impulsive vaccination $\bar{p}=0.5$, as proposed in [12]. Figure 3 shows the optimal evolution (in logarithmic scale) and its phase diagram as found by the algorithm proposed here. The value of the optimal cost function is 190 , and the value of the same cost function for the constant vaccination of [12], with percentage $\bar{p}=0.5$, is 700 (almost $200 \%$ greater). Note the evolution of the susceptible (going to a periodic condition) and the evolution of infected (going to $i^{*}=10^{-5}$ ) and also note that in Figure 3 the susceptibles reach the equilibrium before $T=50$, the half of the optimization horizon.

The control action prescription resulting from the dynamic optimization of impulsive dynamic system models can be implemented efficiently as health policy actions, from the practical point of view. Other computational tests have been performed, with other equilibrium points, and with linear, non-convex and discontinuous cost-functions, with satisfactory results. The technique presented here can be used easily, considering other control actions (such as isolation, or mixed strategies). 


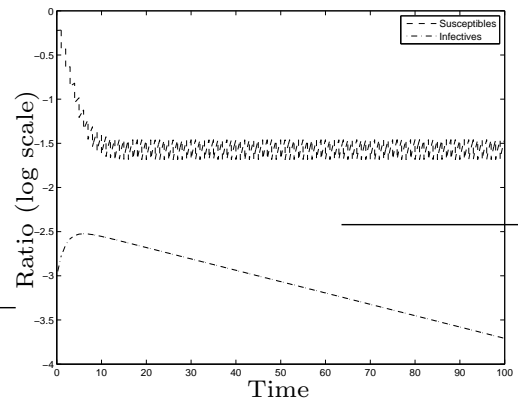

(a) Time-evolution

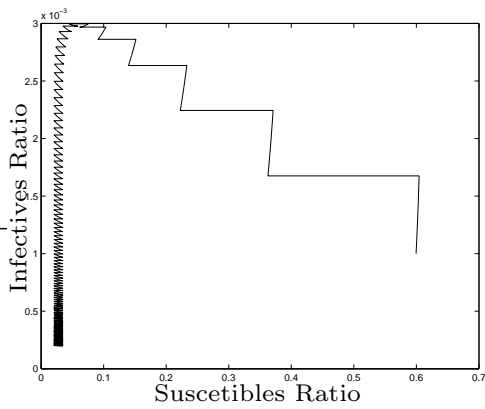

(b) Phase diagram

Figure 2: Behavior of the SIR system (4.4) with constant impulsive vaccination $\bar{p}=0.5$.

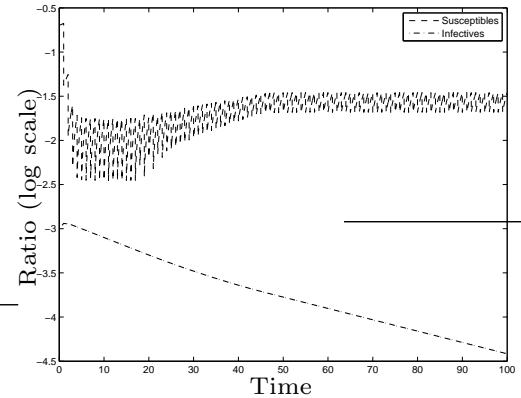

(a) Time-evolution

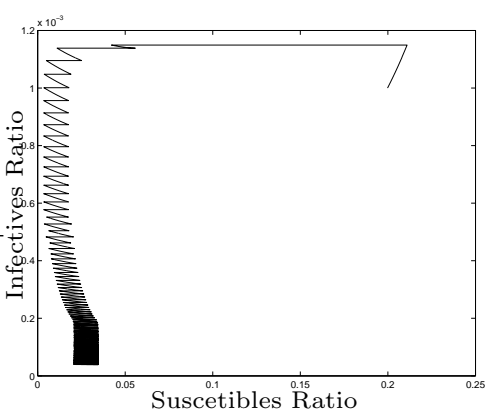

(b) Phase diagram

Figure 3: Behavior of the SIR system (4.4) with the optimal impulsive control action.

\section{Conclusions}

This article has presented an open-loop discrete-time dynamic optimization scheme for impulsive control problems, similar to OLFC. The numerical results obtained in two case studies show the applicability and efficiency of the proposed approach in the context of biological control problems. The methodology is expected to be useful in other contexts of applications in real-world dynamic problems.

Resumo. Este trabalho propõe um método de otimização dinâmica em tempo discreto em malha aberta para problemas de controle impulsivo com variáveis contínuas. Essa metodologia pode ser mais útil que o controle ótimo clássico, uma vez que prescreve ações de controle a serem aplicadas apenas em instantes discretos, nos quais são introduzidas descontinuidades nas variáveis de estado do sistema. Dois estudos de casos são apresentados: o controle biológico de pragas na lavoura usando um modelo presa-predador e o controle de epidemias por vacinação ótima usando um modelo SIR.

Palavras-chave. Controle Impulsivo, Programação Dinâmica, Presa-Predador, SIR. 
Cardoso and Takahashi

\section{Referências}

[1] D.P. Bertsekas, "Dynamic Programming and Optimal Control", Athena Scientific, 1995.

[2] M.S. Branicky, "Studies in Hybrid Systems: Modeling, Analysis, and Control", Phd dissertation, Electrical Engineering and Computer Science Department, Massachusetts Institute of Technology, USA, 1995.

[3] R.T.N. Cardoso, R.H.C. Takahashi, Algoritmos para programação dinâmica baseados em famílias invariantes [in portuguese], Simpósio Brasileiro de Pesquisa Operacional, 2005.

[4] A.C. Chiang, "Elements of Dynamic Optimization", Mc Graw Hill, 1992.

[5] A.R. da Cruz, R.T.N. Cardoso, E.F. Wanner, R.H.C. Takahashi, A multiobjective non-linear dynamic programming approach for optimal biological control in soy farming via NSGA-II, IEEE Congress on Evolutionary Computation, 2007.

[6] A.N. Dar'in, A.B. Kurzhanskii, A.V. Selesznev, The dynamic programming method in impulsive control synthesis, Ordinary Differential Equations, 41, No. 11 (2005), 1491-1500.

[7] A. d'Onofrio, On pulse vaccination strategy in the SIR epidemic model with vertical transmission, Applied Mathematics Letters, 18, No. 7 (2005), 729-732.

[8] C.C. Feltrin, M. Rafikov, Aplicação da função de Lyapunov num problema de controle ótimo de pragas [in portuguese], in "Seleta do XXIV CNMAC" (E.X.L. de Andrade et al., eds.), TEMA - Tendências em Matemática Aplicada e Computacional, Vol. 3, No.2, pp. 83-92, SBMAC, 2002.

[9] H.W. Hetchcote, The mathematics of infectious diseases, SIAM Review, 42, No. 4 (2000), 599-653.

[10] V. Lakshmikantham, D.D. Bainov, P.S. Simeonov, "Theory of Impulsive Differential Equations", Series in Modern Applied Mathematics, World Scientific, Singapore, 1989.

[11] B. Liu, Z. Teng, L. Chen, Analysis of a predator-prey model with Holling II functional response concerning impulsive control strategy, Journal of Computational and Applied Mathematics, 193, No. 2 (2006), 347-362.

[12] E.G. Nepomuceno, "Dinâmica, Modelagem e Controle de Epidemias", Tese de doutorado [in portuguese], Programa de Pós-Graduação em Engenharia Elétrica, Universidade Federal de Minas Gerais, Brasil, 2005.

[13] J.T. Sun, Y.P. Zhang, Stability analysis of impulsive control systems, IEE Proceedings in Control Theory and Applications, 150, No. 4 (2003), 331-334.

[14] T. Yang, Impulsive control, IEEE Transactions on Automatic Control, 44, No. 5 (1999), 1081-1083. 